\title{
Subject Index Vol. 24,1997
}

Infusionstherapie Transfusionsmedizin

A antigen depression 5354 ABO blood group 5354 Acetylsalicitic acid 158, 278 Acute

leukemia 5354

lymphoblastic leukaemia 3138

respiratory failure 5340 Albumin 14

Allogeneic transplantation 6419

Antigens 6412

Antiplatelet therapy 158

Antithrombin III 3138

Aspirin 6428

Autologous blood donation 272

- $\quad$ hemapheresis 272

Bacterial contamination 114

Bleeding time 6428

Blood (plasma glucose, plasma sucrose)

3144 Blood component transfusion 2106

donors 2 78, 3167

group testing 5354 -substitutes 2114

transfusion 120, 2 100, 3167

volume 3132

volume determination 3132

Carbon dioxide production 5340

CD34 6404

CD34+blood cells 6419

CELIA (competitive enzyme-linked

immunoassay) 14 Cell separator MCS 3p 272 Classes of regulatory circuits 3158 Coagulation 2 92 -disorders 3138 Coagulopathy, plasmatic 151 Colorectal cancer prognosis 120 Compatibility testing 2106 Complications (pulmonary edema,

burn) 110 Concentration measurement 14 Congenital disorders of haemostasis:

surgery (prerequisites), replacement

therapy 146 Critical hematocrit 2100 Critically ill 292

DIN EN ISO 9001 3151,3158

Elastase 5348

Emergency transfusion 2106

EN 450013158

Enteral nutrition 5340

Error management 3151

Erythrocyte volume 3132

Ether elution according to Rubin 6410 
- evaporation 6410

External audits 3151

Factor XIII 5348 Fibrinogen 3138 Fibrinolysis 151 Flow cytometry 3 132, 6412 Fresh frozen plasma 3 138, 6397

Global coagulation tests 143 GLP 3158 GMP 3158

Haemostatic disorders 2106

HBV 3167

HCV 3167

Heart (coronary artery disease) 2100

Hematocrit 3132

Hematopoietic progenitor cells 6419

-stem cell 6404

Hemodilution 2100

Hemoglobin modifications 2114

Hemostaseologic differential diagnosis

143 Hemostatic surgical risk 143 Henoch-Schönlein purpura 5348 Heparin 158 HIV 3167

Human albumin 292 -IgG 14

Hydroxyethylstarch 292 Hypothermia 151

Immunmodulation 292 Immunoaffinity selection 6419 Immunomodulation 120, 3171

Indocyanine green 110 Industrial cooperation 3158 Interleukin-6 6404

Intravenous immunoglobulins 3171

L-asparaginase 3138

Low molecular weight heparins 158

Macrocirculation 292 Massive transfusion 2106 Matrix of equivalence 3151 Measurement techniques (extracellular

fluid volume) 3144 Metabolism (glucose, sucrose) 3144 Microcirculation 292 Multicenter study 6397

Neuromuscular disorders (acute therapy and intensive care with intravenous immunoglobulins) 3 171

Partial pressure of carbon dioxide in

arterial blood 5340 Perfluorocarbons 2114 Perioperative period 292 Pharmacokinetics

(glucose, sucrose)

3144 Plasma proteins 110

- $\quad$ volume measurement 110

Plasmin inhibitor deficiency: hereditary,

acquired, clinical symptoms, treatment

286 Platelet disorders 6428

function 6428 --test 278

reduction 6419

storage 6412

Post-transfusion bacterial sepsis 114 Postoperative infection risk 120 Predictive coagulation parameters 139 Preoperative diagnosis of coagulation

139

QM system 3151 Quality assurance 278

Red cell concentrates 282 Regional anesthesia 158 Respiratory minute volume 5340 Routine coagulation screening tests 139 
Spinal hematomas $158 \quad$ Time of elution 6410 Transmission electron microscopy 6412 Stability 6 397 Toxoplasma 282

Stem cell factor 6404 Toxoplasma DNA $282 \quad$ Vasculitis 5348

Storage conditions $6397 \quad$ Transfusion 2114,2 $82 \quad$ von WiUebrand's disease 6428

- reaction 114

Thrombocytes 151 -risks $2106 \quad$ Yersinia enterocolitica 114

450

Subject Index Vol. 24,1997 